\title{
Reduction of Aldehydes and Ketones by Transfer Hydrogenation with 1,4-
}

\section{Butanediol}

\author{
Hannah C. Maytum ${ }^{\mathrm{a}}$, Bahereh Tavassoli ${ }^{\mathrm{b}}$ and Jonathan M. J. Williams ${ }^{\mathrm{a}}$ \\ ${ }^{a}$ Department of Chemistry, University of Bath, Claverton Down, Bath, BA2 7AY, UK \\ ${ }^{b}$ GlaxoSmithKline Research and Development, Old Powder Mills, Tonbridge, Kent, TN11 9AN, UK
}

General Methods: Reactions that required the use of anhydrous, inert atmosphere techniques were carried out under an atmosphere of argon or nitrogen. All reactions were carried out in oven dried, nitrogen (or argon) purged glassware, unless stated otherwise. In all cases, solvents were obtained by passing through anhydrous alumina columns using an Innovative Technology Inc. PS-400-7 solvent purification system. All reagents were purchased from commercial suppliers: Acros Organics, Alfa Aesar, Avocado, Fluka, Lancaster, Sigma Aldrich or Strem, unless preparative details are provided.

TLC using aluminium backed plates precoated with Machery-Nagel Sil G/UV $\mathrm{U}_{254 \mathrm{~nm}}$ neutral silica were used to follow reactions and flash chromatography. Visualisation of TLC plates was by $254 \mathrm{~nm}$ UV light and/or $\mathrm{KMnO}_{4}$ dip followed by gentle warming. Flash chromatography was carried out using Davisil LC $60 \AA$ silica gel (3570 micron) purchased from Fluorochem. Organic layers were routinely dried with $\mathrm{MgSO}_{4}$, and evaporated using a Büchi rotary evaporator, or high vacuum line. Where necessary, further drying was obtained using a high vacuum line.

NMR spectra were routinely run in $\mathrm{CDCl}_{3}$ on either a Bruker Avance $250(250 \mathrm{MHz})$ or a Bruker Avance $300(300 \mathrm{MHz})$ instrument and recorded at the following frequencies: proton $\left({ }^{1} \mathrm{H}-250 / 300 \mathrm{MHz}\right)$ and carbon $\left({ }^{13} \mathrm{C}-62.9 / 75.4 \mathrm{MHz}\right)$. Chemical shifts are reported relative to the residual solvent peak where possible or alternatively to $\mathrm{SiMe}_{4}(\delta=0.00 \mathrm{ppm})$ as the internal standard. Coupling constants $(J)$ are given in $\mathrm{Hz}$ and multiplicities are denoted as: singlet (s), doublet (d), triplet ( $\mathrm{t}$ ), quartet (q), unresolved multiplet (m) or broad (br.). Signals are assigned as general assignments classified as aromatic (Ar), phenyl $(\mathrm{Ph})$, quaternary carbon $(\mathrm{C})$, methyne carbon $(\mathrm{CH})$, methylene carbon $\left(\mathrm{CH}_{2}\right)$ and methyl carbon $\left(\mathrm{CH}_{3}\right)$. Structural assignments of both proton and carbons were achieved with comparisons from analogous literature compounds; references are given in all cases. 
High Pressure Liquid Chromatography (HPLC) was carried out using a PerkinElmer Series 200 and a Chiracel OD ${ }^{\circledR}$ column obtained from Fisher Scientific supplies; the solvent and flow rate used are detailed in the relevant experiment.

\section{Experimental Methods:}

Preparation of $\mathbf{R u}\left(\mathrm{PPh}_{3}\right)_{3}(\mathbf{C O}) \mathbf{H}_{2}$ : To a nitrogen purged, 3-necked round bottomed flask charged with triphenylphosphine (6.28 g, $23.9 \mathrm{mmol})$ was added degassed anhydrous methanol $(200 \mathrm{~mL})$. The mixture was heated at reflux for 10 minutes, forming a solution. In quick succession, ruthenium trichloride hydrate $(1.04 \mathrm{~g}, 4.0$ $\mathrm{mmol})$ in methanol $(40 \mathrm{~mL})$, aqueous formaldehyde $(37 \% \mathrm{w} / \mathrm{w})(40 \mathrm{~mL})$ and potassium hydroxide $(1.20 \mathrm{~g}, 21.4 \mathrm{mmol})$ in methanol $(40 \mathrm{~mL})$ were added. The resulting solution was heated for 30 minutes at reflux and then cooled in an ice bath with stirring for a further 30 minutes. The grey precipitate was collected by vacuum filtration and washed with absolute ethanol $(50 \mathrm{~mL})$, water $(50 \mathrm{~mL})$, absolute ethanol $(50 \mathrm{~mL})$ and finally hexane $(50 \mathrm{~mL})$. The crude product was dissolved in toluene and filtered through a column of neutral alumina and washed through thoroughly with toluene. The toluene solution was concentrated in vacuo to approximately $20 \mathrm{~mL}$ and layered with anhydrous methanol producing a precipitate which was collected by vacuum filtration as a white solid $\left(\mathrm{Ru}\left(\mathrm{PPh}_{3}\right)_{3}(\mathrm{CO}) \mathrm{H}_{2}\right)(2.50 \mathrm{~g}, 68 \%)$.

Representative procedure for the reduction of propiophenone with different diols and alcohols: To oven dried and nitrogen purged Radleys carousel tubes containing $\mathrm{Ru}\left(\mathrm{PPh}_{3}\right)_{3}(\mathrm{CO}) \mathrm{H}_{2}$ (22.9 mg, $0.025 \mathrm{mmol}, 0.025$ equiv.), was added propiophenone $3\left(0.1342 \mathrm{~g}, 1 \mathrm{mmol}, \rho=1.009 \mathrm{gmL}^{-1}, 0.1330 \mathrm{~mL}, 1\right.$ equiv.) and the required hydrogen donor ( $1 \mathrm{mmol}, 1$ equiv.) followed by toluene $(1 \mathrm{~mL})$. The reactions were heated to reflux for 24 hours and were then filtered through Celite and silica, washed through with DCM and concentrated in vacuo. Conversions were calculated from peak integral ratios characteristic of propiophenone $\mathbf{3}$ and 1-phenyl-1propanol 4 in the crude ${ }^{1} \mathrm{H}$ NMR. Crude products were not isolated.

\section{Representative procedure for the reduction of other carbonyl compounds using} 1,4-butanediol, ligand and base: To oven dried and nitrogen purged Radleys 
carousel tubes containing $\mathrm{Ru}\left(\mathrm{PPh}_{3}\right)_{3}(\mathrm{CO}) \mathrm{H}_{2}$ (22.9 mg, $0.025 \mathrm{mmol}, 0.025$ equiv.), DPE-phos (13.5 mg, $0.025 \mathrm{mmol}, 0,025$ equiv.), $\mathrm{KO}^{t} \mathrm{Bu}(5.6 \mathrm{mg}, 0.05 \mathrm{mmol}, 0.05$ equiv.), was added the required substrate ( $1 \mathrm{mmol}, 1$ equiv.) and 1,4-butanediol ( $0.092 \mathrm{~g}, 1 \mathrm{mmol}, \rho=1.017 \mathrm{gmL}^{-1}, 0.089 \mathrm{~mL}, 1$ equiv.) followed by toluene $(1 \mathrm{~mL})$. The reactions were heated to reflux for 24/48 hours. The reactions were then filtered through Celite and silica, washed through with DCM and concentrated in vacuo. Conversions were calculated from peak integral ratios characteristic of the required substrates and their corresponding alcohols in the crude ${ }^{1} \mathrm{H}$ NMR.<smiles>OC1CCCc2ccccc21</smiles>

$\alpha$-Tetralol ${ }^{[1]}$ (Entry 1, Table 2): According to the representative procedure, using $\alpha$ Tetralone (0.1462 g, $1 \mathrm{mmol}, \rho=1.099 \mathrm{gmL}^{-1}, 0.1330 \mathrm{~mL}, 1$ equiv.) the title compound was obtained in $91 \%$ conversion after 48 hours. The product was not isolated; conversion was calculated from peak integral ratios characteristic of $\alpha$ Tetralol in the crude ${ }^{1} \mathrm{H}$ NMR spectrum. ${ }^{1} \mathrm{H}$ NMR $\left(250 \mathrm{MHz}, \mathrm{CDCl}_{3}, 25{ }^{\circ} \mathrm{C}\right): \delta=1.76$ - 2.08 (m, 4H), 2.78 - 2.94 (m, 2H), 4.82 (m, 1H, -Cㅌ-OH), 7.13 - 7.39 (m, 4H, Ph$\underline{\mathrm{H}})$.<smiles>COc1ccc(C(C)O)cc1</smiles>

1-(4-Methoxyphenyl)ethanol ${ }^{[2]}$ (Entry 2, Table 2): According to the representative procedure, using $p$-methoxyacetophenone $(0.1502 \mathrm{~g}, 1 \mathrm{mmol}, 1$ equiv.) the title compound was obtained in $87 \%$ conversion after 24 hours. The product was not isolated; conversion was calculated from peak integral ratios characteristic of 1-(4Methoxyphenyl)ethanol in the crude ${ }^{1} \mathrm{H}$ NMR spectrum. ${ }^{1} \mathrm{H}$ NMR $\left(250 \mathrm{MHz}, \mathrm{CDCl}_{3}\right.$, $\left.25^{\circ} \mathrm{C}\right): \delta=1.19\left(\mathrm{~d}, 3 \mathrm{H}, J=6.4 \mathrm{~Hz},-\underline{C H}_{3}\right), 3.69\left(\mathrm{~s}, 3 \mathrm{H},-\mathrm{OC}_{3}\right), 4.73(\mathrm{q}, 1 \mathrm{H}, J=6.4$ $\mathrm{Hz},-\mathrm{C} \underline{\mathrm{H}}-\mathrm{OH}), 6.78$ (d, 2H, $J=8.8 \mathrm{~Hz}, \mathrm{Ph}-\underline{\mathrm{H}}), 7.08$ (d, 2H, J=7.1 Hz, Ph- $\underline{\mathrm{H}}$ ).<smiles>CC(O)c1cccc(Cl)c1</smiles> 
1-(3-Chlorophenyl)ethanol ${ }^{[3]}$ (Entry 3, Table 2): According to the representative procedure, using 3'-chloroacetophenone $\left(0.1546 \mathrm{~g}, 1 \mathrm{mmol}, \rho=1.191 \mathrm{gmL}^{-1}, 0.1230\right.$ $\mathrm{mL}, 1$ equiv.) the title compound was obtained and purified by column chromatography $\left(1: 1\right.$ petroleum ether (b.p. $\left.40-60{ }^{\circ} \mathrm{C}\right) /$ diethyl ether, $\left.\mathrm{R}_{\mathrm{f}}=0.32\right)$ afforded the title compound as a pale yellow liquid (0.1240 g, 79\%). ${ }^{1} \mathrm{H}$ NMR (300 $\left.\mathrm{MHz}, \mathrm{CDCl}_{3}, 25^{\circ} \mathrm{C}\right): \delta=1.38$ (d, $\left.3 \mathrm{H}, J=6.5 \mathrm{~Hz},-\underline{\mathrm{C}}_{3}\right), 1.90$ (br s, $\left.1 \mathrm{H},-\mathrm{O} \underline{\mathrm{H}}\right), 4.77$ (q, $1 \mathrm{H}, J=3.3,9.9 \mathrm{~Hz},-\mathrm{C} \underline{\mathrm{H}}(\mathrm{OH})-), 7.12-7.17$ (m, 4H, Ar- $\underline{\mathrm{H}}) .{ }^{13} \mathrm{C} \mathrm{NMR}(75 \mathrm{MHz}$, $\left.\mathrm{CDCl}_{3}, 25^{\circ} \mathrm{C}\right): \delta=25.2,69.8,123.5,125.6,127.5,129.8,134.3,147.8$.

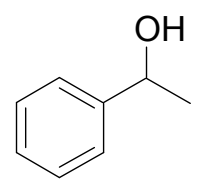

sec-Phenethyl alcohol ${ }^{[4]}$ (Entry 4, Table 2): According to the representative procedure, using acetophenone $\left(0.1202 \mathrm{~g}, 1 \mathrm{mmol}, \rho=1.030 \mathrm{gmL}^{-1}, 0.1200 \mathrm{~mL}, 1\right.$ equiv.) the title compound was obtained and purified by column chromatography $(7: 3$ petroleum ether (b.p. $40-60{ }^{\circ} \mathrm{C}$ )/diethyl ether, $\mathrm{R}_{\mathrm{f}}=0.21$ ) afforded the title compound as a pale yellow liquid $(0.1027 \mathrm{~g}, 84 \%) .{ }^{1} \mathrm{H}$ NMR $\left(300 \mathrm{MHz}, \mathrm{CDCl}_{3}, 25{ }^{\circ} \mathrm{C}\right): \delta=1.40$ (d, $\left.2 \mathrm{H}, J=6.5 \mathrm{~Hz},-\underline{\mathrm{CH}}_{3}\right), 1.78$ (br s, $\left.1 \mathrm{H},-\mathrm{OH}\right), 4.79$ (q, $1 \mathrm{H}, J=6.5,19.4 \mathrm{~Hz},-$ $\mathrm{C} \underline{\mathrm{H}}(\mathrm{OH})-), 7.14-7.26$ (m, 5H, Ph- $\underline{\mathrm{H}}) .{ }^{13} \mathrm{C} \mathrm{NMR}\left(75 \mathrm{MHz}, \mathrm{CDCl}_{3}, 25{ }^{\circ} \mathrm{C}\right): \delta=25.1$, $70.4,125.3,127.4,127.7,128.2,128.5,145.8$.

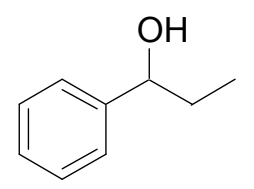

1-Phenyl-1-propanol $4^{[5]}$ (Entry 5, Table 2): According to the representative procedure, using propiophenone $3\left(0.1342 \mathrm{~g}, 1 \mathrm{mmol}, \rho=1.009 \mathrm{gmL}^{-1}, 0.1330 \mathrm{~mL}, 1\right.$ equiv.) the title compound was obtained and purified by column chromatography $4: 1$ petroleum ether (b.p. $40-60{ }^{\circ} \mathrm{C}$ )/diethyl ether, $\mathrm{R}_{\mathrm{f}}=0.17$ ) afforded the title compound as a pale brown liquid $(0.1196 \mathrm{~g}, 88 \%) .{ }^{1} \mathrm{H}$ NMR $\left(300 \mathrm{MHz}, \mathrm{CDCl}_{3}, 25{ }^{\circ} \mathrm{C}\right): \delta=0.85$ (t, 3H, $\left.J=7.4 \mathrm{~Hz},-\underline{\mathrm{C}}_{3}\right), 1.67-1.83\left(\mathrm{~m}, 2 \mathrm{H},-\underline{\mathrm{C}}_{2}-\right), 1.76$ (br s, 1H, -Oㅡㅜ), 4.53 (t, $3 \mathrm{H}, J=6.6 \mathrm{~Hz},-\mathrm{C} \underline{\mathrm{H}}-), 7.18-7.28(\mathrm{~m}, 5 \mathrm{H}, \mathrm{Ph}-\underline{\mathrm{H}}) .{ }^{13} \mathrm{C} \mathrm{NMR}\left(75 \mathrm{MHz}, \mathrm{CDCl}_{3}, 25\right.$ $\left.{ }^{\circ} \mathrm{C}\right): \delta=10.1,31.9,76.0,125.9,127.5,128.4,144.5$. 


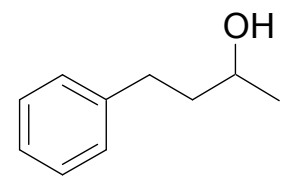

4-Phenyl-2-butanol ${ }^{[6]}$ (Entry 6, Table 2): According to the representative procedure, using 4-phenyl-2-butanone (0.1482 g, $1 \mathrm{mmol}, \rho=0.989 \mathrm{gmL}^{-1}, 0.1499 \mathrm{~mL}, 1$ equiv.) the title compound was obtained and purified by column chromatography $(3: 2$ petroleum ether (b.p. $40-60{ }^{\circ} \mathrm{C}$ )/diethyl ether, $\mathrm{R}_{\mathrm{f}}=0.24$ ) afforded the title compound as a pale yellow liquid $(0.1213 \mathrm{~g}, 92 \%) .{ }^{1} \mathrm{H}$ NMR $\left(300 \mathrm{MHz}, \mathrm{CDCl}_{3}, 25{ }^{\circ} \mathrm{C}\right): \delta=1.28$ (d, 3H, $J=6.2 \mathrm{~Hz},-\underline{\mathrm{CH}}_{3}$ ), 1.47 (br s, $1 \mathrm{H},-\mathrm{O} \underline{\mathrm{H}}$ ), $1.78-1.87$ (m, 2H, $-\underline{\mathrm{C}}_{2}-\mathrm{CH}_{2}$ ), 2.70 $-2.82\left(\mathrm{~m}, 2 \mathrm{H},-\mathrm{CH}_{2}-\underline{\mathrm{C}}_{2}-\right), 3.87-3.90(\mathrm{~m}, 1 \mathrm{H},-\mathrm{CH}(\mathrm{OH})), 7.22-7.37$ (m, 5H, Ph$\underline{\mathrm{H}}) .{ }^{13} \mathrm{C} \mathrm{NMR}\left(75 \mathrm{MHz}, \mathrm{CDCl}_{3}, 25{ }^{\circ} \mathrm{C}\right): \delta=23.6,32.1,40.8,67.5,125.8,128.4$, 142.0.

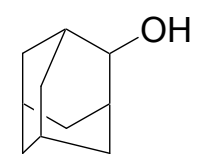

2-Adamantanol ${ }^{[7]}$ (Entry 7, Table 2): According to the representative procedure, using 2-adamantanone $(0.1502 \mathrm{~g}, 1 \mathrm{mmol}, 1$ equiv. $)$ the title compound was obtained in $100 \%$ conversion after 48 hours. The product was not isolated; conversion was calculated from peak integral ratios characteristic of 2-adamantanol in the crude ${ }^{1} \mathrm{H}$ NMR spectrum. ${ }^{1} \mathrm{H}$ NMR $\left(300 \mathrm{MHz}, \mathrm{CDCl}_{3}, 25^{\circ} \mathrm{C}\right): \delta=1.53(\mathrm{~m}, 2 \mathrm{H}), 1.64-1.94$ (m, 11H), 2.07 (m, 2H), 3.88 (s, 1H, - $\underline{\mathrm{H}}(\mathrm{OH})-$ ).<smiles>OC1CCCCC1</smiles>

Cyclohexanol $^{[8]}$ (Entry 8, Table 2): According to the representative procedure, using cyclohexanone $\left(0.098 \mathrm{~g}, 1 \mathrm{mmol}, \rho=0.947 \mathrm{gmL}^{-1}, 0.1036 \mathrm{~mL}, 1\right.$ equiv.) the title compound was obtained and purified by column chromatography $(1: 1$ petroleum ether (b.p. $40-60{ }^{\circ} \mathrm{C}$ )/diethyl ether, $\mathrm{R}_{\mathrm{f}}=0.38$ ) afforded the title compound as a colourless liquid (0.605 g, 60\%). ${ }^{1} \mathrm{H} \mathrm{NMR}\left(300 \mathrm{MHz}, \mathrm{CDCl}_{3}, 25{ }^{\circ} \mathrm{C}\right): \delta=1.06-1.38(\mathrm{~m}, 4 \mathrm{H})$, $1.48-1.94(\mathrm{~m}, 7 \mathrm{H}$, including $-\mathrm{O} \underline{\mathrm{H}}), 3.50-3.70(\mathrm{~m}, 1 \mathrm{H},-\mathrm{C} \underline{\mathrm{H}}(\mathrm{OH})-)$. 


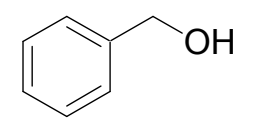

Benzyl alcohol ${ }^{[9]}$ (Entry 9, Table 2): According to the representative procedure, using benzaldehyde $\left(0.1061 \mathrm{~g}, 1 \mathrm{mmol}, \rho=1.044 \mathrm{gmL}^{-1}, 0.1016 \mathrm{~mL}, 1\right.$ equiv.) the title compound was obtained and purified by column chromatography (70:30 petroleum ether (b.p. $40-60{ }^{\circ} \mathrm{C}$ )/diethyl ether, $\mathrm{R}_{\mathrm{f}}=0.32$ ) afforded the title compound as a pale yellow liquid (0.0878 g, 81\%). ${ }^{1} \mathrm{H}$ NMR $\left(300 \mathrm{MHz}, \mathrm{CDCl}_{3}, 25{ }^{\circ} \mathrm{C}\right): \delta=1.82$ (br s, $1 \mathrm{H},-\mathrm{O} \underline{\mathrm{H}}), 4.59$ (s, 2H, $\left.-\underline{\mathrm{C}}_{2}-\mathrm{OH}\right), 7.16-7.31(\mathrm{~m}, 5 \mathrm{H}, \mathrm{Ph}-\underline{\mathrm{H}}) .{ }^{13} \mathrm{C}$ NMR $(75$ $\left.\mathrm{MHz}, \mathrm{CDCl}_{3}, 25^{\circ} \mathrm{C}\right): \delta=65.3,65.8,127.0,127.6,127.8,128.5,140.8$.

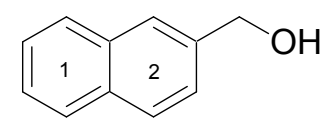

2-Naphthalenemethanol ${ }^{[10]}$ (Entry 10, Table 2): According to the representative procedure, using 2-naphthaldehyde $(0.1562 \mathrm{~g}, 1 \mathrm{mmol}, 1$ equiv. $)$ the title compound was obtained and purified by column chromatography (1:1 petroleum ether (b.p. 40$\left.60{ }^{\circ} \mathrm{C}\right)$ /diethyl ether, $\left.\mathrm{R}_{\mathrm{f}}=0.41\right)$ afforded the title compound as a white solid $(0.1293$ g, 82\%). ${ }^{1} \mathrm{H}$ NMR $\left(300 \mathrm{MHz}, \mathrm{CDCl}_{3}, 25{ }^{\circ} \mathrm{C}\right): \delta=1.85$ (br s, $\left.1 \mathrm{H},-\mathrm{OH}\right), 4.85(\mathrm{~d}, 2 \mathrm{H}, J$ $\left.=4.6 \mathrm{~Hz},-\underline{\mathrm{C}}_{2}-\right), 7.47-7.52\left(\mathrm{~m}, 3 \mathrm{H}, \mathrm{Ar}_{2}-\underline{\mathrm{H}}\right), 7.82-7.87\left(\mathrm{~m}, 4 \mathrm{H}, \mathrm{Ar}_{1}-\underline{\mathrm{H}}\right) .{ }^{13} \mathrm{C} \mathrm{NMR}$ $\left(75 \mathrm{MHz}, \mathrm{CDCl}_{3}, 25^{\circ} \mathrm{C}\right): \delta=65.5,125.1,125.4,125.9,126.2,127.7,127.9,128.3$, 132.9, 133.3, 138.2.

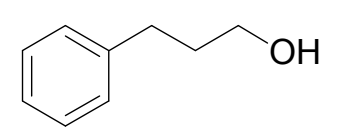

3-Phenyl-1-propanol ${ }^{[11]}$ (Entry 11, Table 2): According to the representative procedure, using cinnamaldehyde $\left(0.1342 \mathrm{~g}, 1 \mathrm{mmol}, \rho=1.019 \mathrm{gmL}^{-1}, 0.1317 \mathrm{~mL}, 1\right.$ equiv.) the title compound was obtained and purified by column chromatography $(3: 2$ petroleum ether (b.p. $40-60{ }^{\circ} \mathrm{C}$ )/diethyl ether, $\mathrm{R}_{\mathrm{f}}=0.24$ ) afforded the title compound as a pale yellow liquid (0.1213 g, 87\%). ${ }^{1} \mathrm{H}$ NMR $\left(300 \mathrm{MHz}, \mathrm{CDCl}_{3}, 25{ }^{\circ} \mathrm{C}\right): \delta=1.54$ (br s, $1 \mathrm{H},-\mathrm{O} \underline{\mathrm{H}}), 1.90-1.98\left(\mathrm{~m}, 2 \mathrm{H},-\mathrm{CH}_{2}-\mathrm{C}_{2}-\mathrm{CH}_{2}-\right), 2.77$ (t, $2 \mathrm{H}, J=7.7 \mathrm{~Hz},-\underline{\mathrm{C}}_{2}-$ $\left.\mathrm{CH}_{2}-\mathrm{CH}_{2} \mathrm{OH}\right) 3.73$ (t, 2H, J=6.4 Hz, $\left.-\mathrm{CH}_{2}-\mathrm{CH}_{2}-\mathrm{CH}_{2} \mathrm{OH}\right), 7.22-7.37$ (m, 5H, Ph- $\underline{\mathrm{H}}$ ). ${ }^{13} \mathrm{C} \mathrm{NMR}\left(75 \mathrm{MHz}, \mathrm{CDCl}_{3}, 25^{\circ} \mathrm{C}\right): \delta=32.0,34.2,62.3,125.8,128.4,128.4,141.8$.

Procedure for the combined use of 1,4-butanediol with methyl levulinate: To an oven dried and nitrogen purged Schlenk tube containing $\mathrm{Ru}\left(\mathrm{PPh}_{3}\right)_{3}(\mathrm{CO}) \mathrm{H}_{2}(22.9 \mathrm{mg}$, 
0.025 mmol, 0.025 equiv.), DPE-phos (13.5 mg, 0.025 mmol, 0,025 equiv.), $\mathrm{KO}^{t} \mathrm{Bu}$ (5.6 mg, $0.05 \mathrm{mmol}, 0.05$ equiv.), was added 1,4-butanediol (0.092 g, $1 \mathrm{mmol}, \rho=$ $1.017 \mathrm{gmL}^{-1}, 0.089 \mathrm{~mL}, 1$ equiv.) and methyl levulinate $(0.1301 \mathrm{~g}, 1 \mathrm{mmol}, \rho=1.051$ $\mathrm{gmL}^{-1}, 0.1238 \mathrm{~mL}, 1$ equiv.) followed by toluene $(1 \mathrm{~mL})$. The reactions were heated to reflux for 24 hours. The reaction was then filtered through Celite and silica, washed through with DCM and concentrated in vacuo. Conversion was calculated by the peak integral ratio characteristic of both $\gamma$-butyrolactone 5 and $\gamma$-valerolactone $\mathbf{6}$ in the crude ${ }^{1} \mathrm{H}$ NMR spectrum.

\section{Procedure for the transfer hydrogenation with Noyori catalyst using iso-} propanol: To an oven dried and argon purged $250 \mathrm{~mL}$ round bottomed flask containing $\left[\mathrm{Ru}(p \text {-cymene }) \mathrm{Cl}_{2}\right]_{2}(6.1 \mathrm{mg}, 0.01 \mathrm{mmol}, 0.001$ equiv. $)$ and $(S, S)$-TsDPEN (14.7 mg, $0.04 \mathrm{mmol}, 0.004$ equiv.) was added iso-propanol $(5 \mathrm{~mL})$. The reaction was heated to $80{ }^{\circ} \mathrm{C}$ for 1 hour under an atmosphere of nitrogen. A pale orange solution was obtained. Acetophenone $\left(1.202 \mathrm{~g}, 10 \mathrm{mmol}, \rho=1.030 \mathrm{gmL}^{-1}, 1.1700 \mathrm{~mL}, 1\right.$ equiv.) and iso-propanol (94 $\mathrm{mL})$ were degassed separately under argon and then added to the pale orange solution (once it had been allowed to cool to room temperature). A solution of $0.1 \mathrm{M} \mathrm{KOH}$ in iso-propanol was then prepared $(0.056 \mathrm{~g}$ $\mathrm{KOH}$ in $10 \mathrm{~mL}$ iso-propanol) and $2 \mathrm{~mL}$ of this solution was added to the pale orange solution, upon which a pale pink solution was obtained which turned orange again once stirring was restored. The reaction was then stirred at room temperature for 14 hours. After this time, the reaction was quenched with $2 \mathrm{M} \mathrm{HCl}(1 \mathrm{~mL})$ and stirred for a further 30 minutes at room temperature. The reaction mixture was then concentrated in vacuo, and ethyl acetate was added. The ethyl acetate layer was then washed with saturated brine solution three times, dried over $\mathrm{MgSO}_{4}$, filtered and reduced in vacuo giving a pale yellow liquid (ee $=98 \%$, Chiracel OD column 90:10 hexane:isopropanol solvent, $0.5 \mathrm{~mL} / \mathrm{min}$ flow, retention times $=9.7 \mathrm{~min}(\mathrm{~S}), 12.3 \mathrm{~min}(R)$ ). Conversion was calculated by analysis of the crude product ${ }^{1} \mathrm{H}$ NMR spectrum using the characteristic peaks of acetophenone and $(S)$-sec-phenethyl alcohol.

\section{Procedure for the transfer hydrogenation with Noyori catalyst using 1,4-} butanediol and acetophenone: To an oven dried and argon purged $10 \mathrm{~mL}$ round bottomed flask containing $\left[\mathrm{Ru}(p \text {-cymene }) \mathrm{Cl}_{2}\right]_{2}(6.1 \mathrm{mg}, 0.01 \mathrm{mmol}, 0.001$ equiv. $)$ and $(S, S)$-TsDPEN (14.7 mg, 0.04 mmol, 0.004 equiv.) was added 1,4-butanediol (0.4506 
g, $5 \mathrm{mmol}, \rho=1.017 \mathrm{gmL}^{-1}, 0.443 \mathrm{~mL}, 0.5$ equiv.). The reaction mixture was then heated to $80{ }^{\circ} \mathrm{C}$ for 1 hour under an atmosphere of nitrogen. Meanwhile, $\mathrm{KOH}(56.0$ $\mathrm{mg}, 1 \mathrm{mmol}, 0.1$ equiv.) was dissolved in 1,4-butanediol $(0.4506 \mathrm{~g}, 5 \mathrm{mmol}, \rho=1.017$ $\mathrm{gmL}^{-1}, 0.443 \mathrm{~mL}, 0.5$ equiv.) by stirring at room temperature. Once the reaction mixture had cooled to room temperature, the base mixture was added, followed by acetophenone (1.202 g, $10 \mathrm{mmol}, \rho=1.030 \mathrm{gmL}^{-1}, 1.1700 \mathrm{~mL}, 1$ equiv.). The reaction was then stirred at room temperature for 72 hours. After this time the reaction was quenched with $2 \mathrm{M} \mathrm{HCl}$ and stirred for a further 30 minutes at room temperature. Ethyl acetate was then added, and the organic layer was washed with brine three times, dried over $\mathrm{MgSO}_{4}$, filtered and reduced in vacuo giving a pale yellow liquid (ee $=98 \%$, Chiracel OD column 90:10 hexane:iso-propanol solvent, $0.5 \mathrm{~mL} / \mathrm{min}$ flow, retention times $=9.7 \mathrm{~min}(\mathrm{~S}), 12.3 \mathrm{~min}(R))$. Conversion was calculated by analysis of the crude product ${ }^{1} \mathrm{H}$ NMR spectrum using the characteristic peaks of acetophenone and $(S)$-sec-phenethyl alcohol.

Procedure for the transfer hydrogenation with Noyori catalyst with 1,4butanediol and 3'-chloroacteophenone: To an oven dried and argon purged Young's Tap NMR tube containing $\left[\mathrm{Ru}(p \text {-cymene }) \mathrm{Cl}_{2}\right]_{2}(6.1 \mathrm{mg}, 0.01 \mathrm{mmol}, 0.001$ equiv.), (S,S)-TsDPEN 11 (14.7 mg, $0.04 \mathrm{mmol}, 0.004$ equiv.) and $\mathrm{KOH}$ (4.5 mg, $0.08 \mathrm{mmol}, 0.008$ equiv. $)$ were added 3'-chloroacetophenone $(0.1546 \mathrm{~g}, 1 \mathrm{mmol}, \quad \rho=$ $1.191 \mathrm{gmL}^{-1}, 0.1230 \mathrm{~mL}, 1$ equiv.) and 1,4-butanediol (0.0901 g, $1 \mathrm{mmol}, \rho=1.017$ $\mathrm{gmL}^{-1}, 0.090 \mathrm{~mL}, 1$ equiv.). The reaction was heated to $40{ }^{\circ} \mathrm{C}$ for 24 hours (with intermittent shaking as a means of stirring). After this time the reaction was quenched with $2 \mathrm{M} \mathrm{HCl}$ and stirred for a further 30 minutes at room temperature. Ethyl acetate was then added, and the organic layer was washed with brine three times, dried over $\mathrm{MgSO}_{4}$, filtered and reduced in vacuo giving a pale yellow liquid (ee $=82 \%$, Chiracel OD column 90:10 hexane:iso-propanol solvent, $0.5 \mathrm{~mL} / \mathrm{min}$ flow, retention times $=$ $9.3 \mathrm{~min}(\mathrm{~S}), 10.8 \mathrm{~min}(R)$ ). Conversion was calculated by analysis of the crude product ${ }^{1} \mathrm{H}$ NMR spectrum using the characteristic peaks of 3 '-chloroacetophenone and 1-(3-Chlorophenyl)ethanol.

\section{References:}

[1] K. Ishihara, M. Hantano and S. Suzuki, J. Am. Chem. Soc. 2006, 128, 9998-9999. 
[2] G. V. M. Sharma, P. S. Lakshmi, A. C. Kunwar, R. Ravi and K. L. Reddy, J. Org. Chem. 2005, 71, 3967-3969.

[3] C. A. Kingsbury and C. H. Wang, J. Org. Chem., 1972, 37, 15, 2489 - 2494.

[4] P. Renaud, J. Cossy, P. I. Dalko, C. Cadot, R. Chuard and C. Ollivier, J. Org. Chem., 2002, 67, $7193-7202$.

[5] S. Bräse and S. Dahmen, Chem. Comm. 2002, 26-27.

[6] Y-Q. Tu, C-A. Fan, L. Shi, M. Wang, W-J. Xia, F-M. Zhang and Y-M. Zhoa, J. Am. Chem. Soc., 2005, 127, 10836 - 10837.

[7] F. W. van Deursen and P.K. Korver, Tetrahedron Lett., 1967, 40, 3923 - 3928.

[8] B. Figadère, A. Cave, C. Chaboche, X. Franck and J-F. Peyrat, J. Org. Chem., 1994, 59, $7138-7141$.

[9] H-J. Ziu, J-X. Jiang, L-C. Li, C. U. Pittman Jr., J. Ren and Y. Ren, Eur. J. Org. Chem., 2006, $1981-1990$.

[10] A. Solladié-Cavallo and A. Diep-Vohuule, J. Org. Chem., 1995, 60, 3494 3498.

[11] T. Le Gall, C. Mioskowski and J-P. Goddard, Org. Lett. 2000, 2, 10, 1455-1456. 


\section{NMR Spectra:}<smiles>OC1CCCc2ccccc21</smiles>

Entry 1, Table 2

(crude ${ }^{1} \mathrm{H}$ NMR spectra, contains $\alpha$-tetralol, $\alpha$-tetralone, 1,4-butanediol, $\gamma$-butyrolactone and residual solvents)

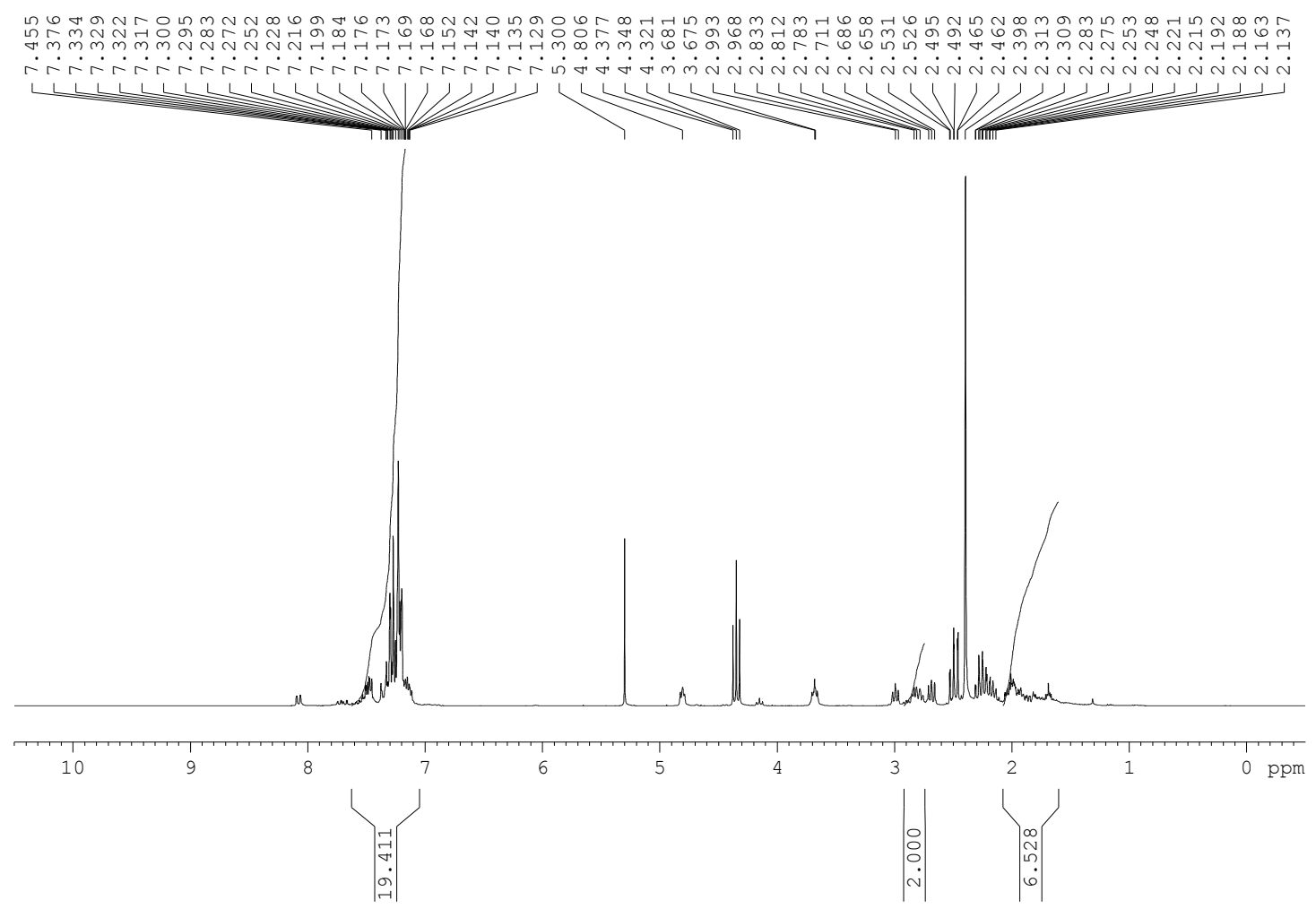


<smiles>COc1ccc(C(C)O)cc1</smiles>

Entry 2, Table 2

(crude ${ }^{1} \mathrm{H}$ NMR spectra, contains 1-(4-methoxyphenyl)ethanol, $p$-methoxyacetophenone, 1,4butanediol, $\gamma$-butyrolactone and residual solvents)

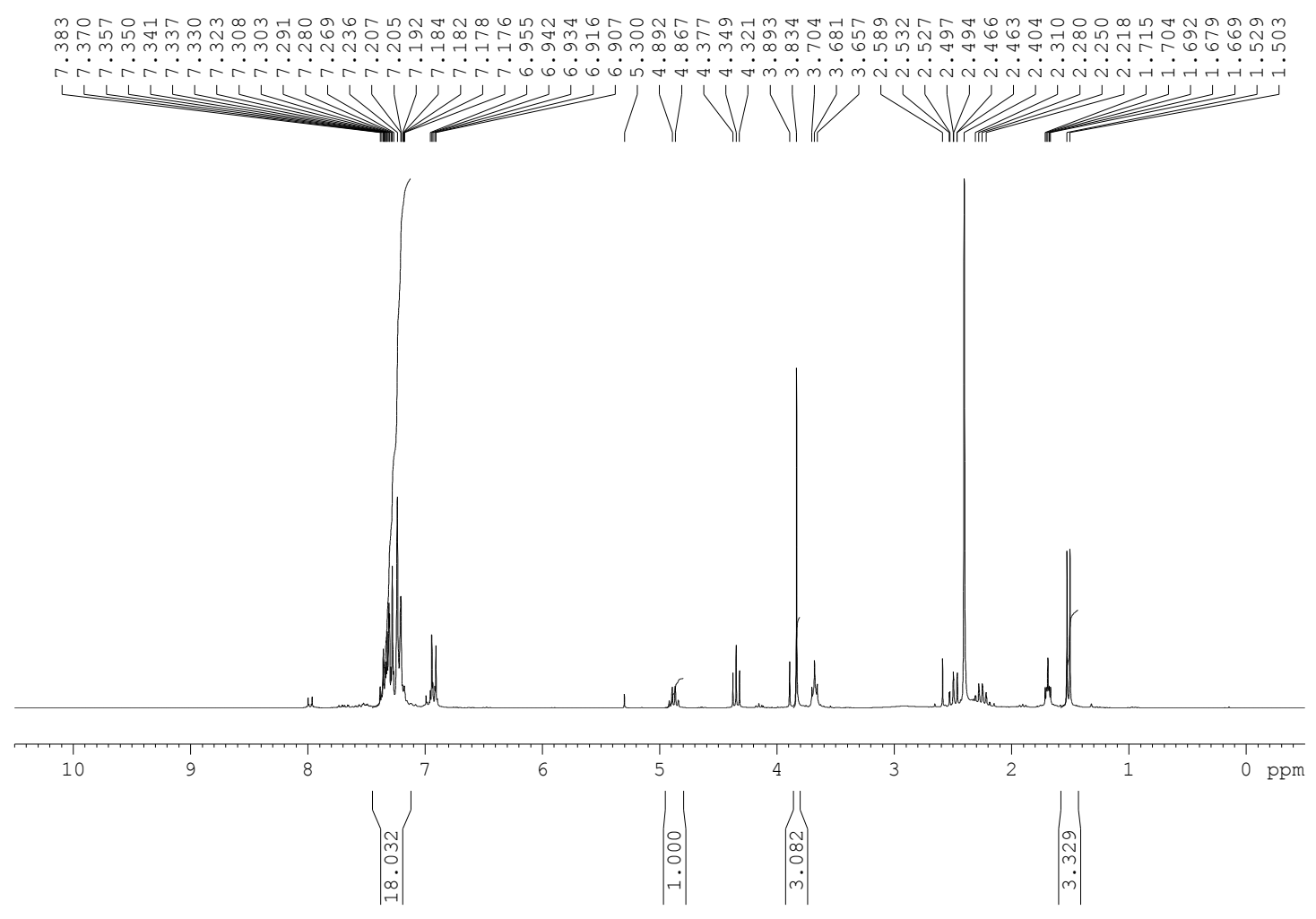




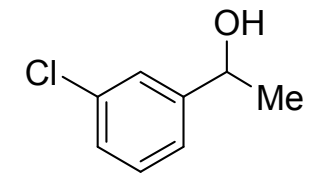

Entry 3, Table 2

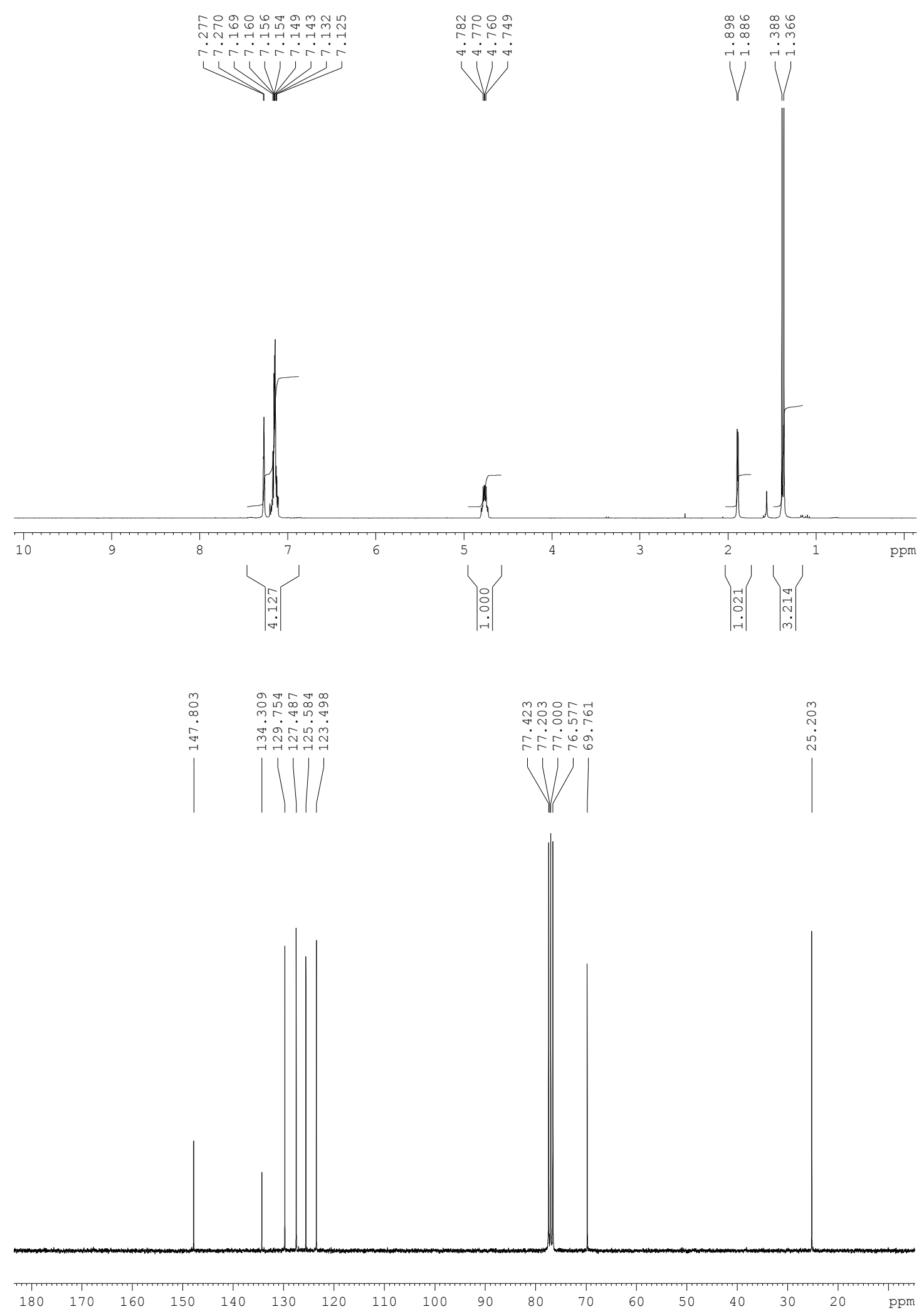


<smiles>CC(O)c1ccccc1</smiles>

Entry 4, Table 2
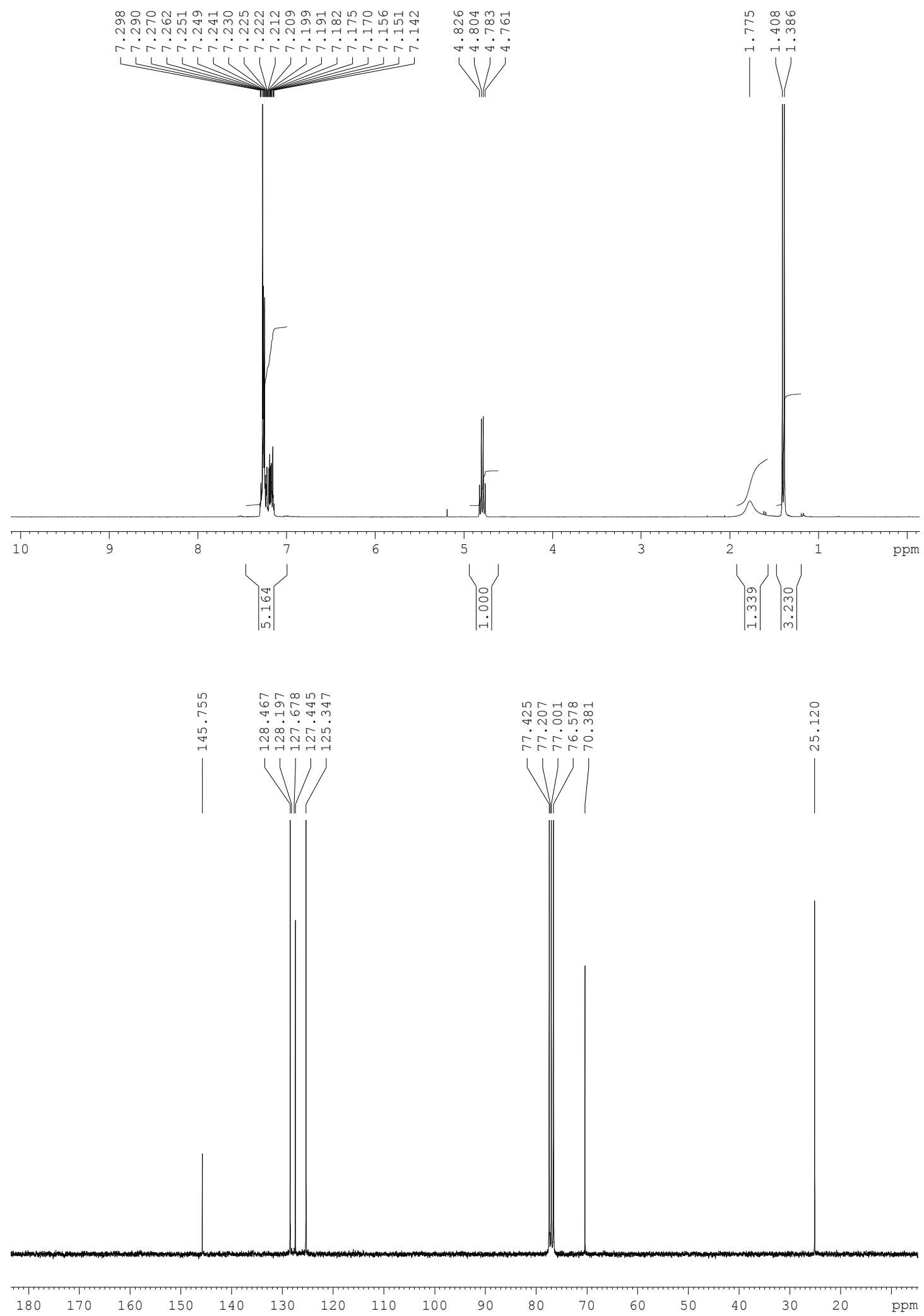
<smiles>CCC(O)c1ccccc1</smiles>

\section{Entry 5, Table 2}

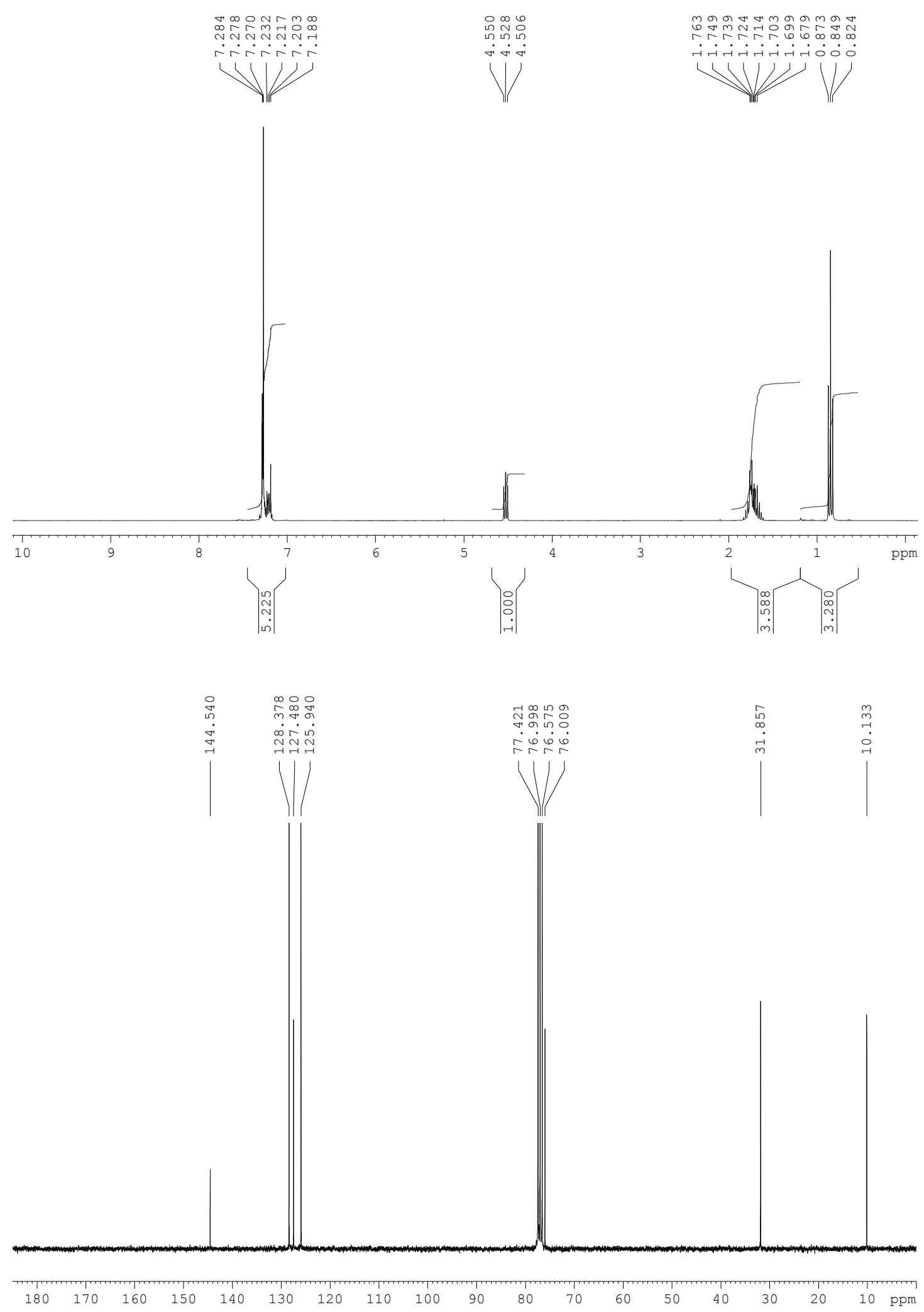


<smiles>CC(O)CCc1ccccc1</smiles>

\section{Entry 6, Table 2}

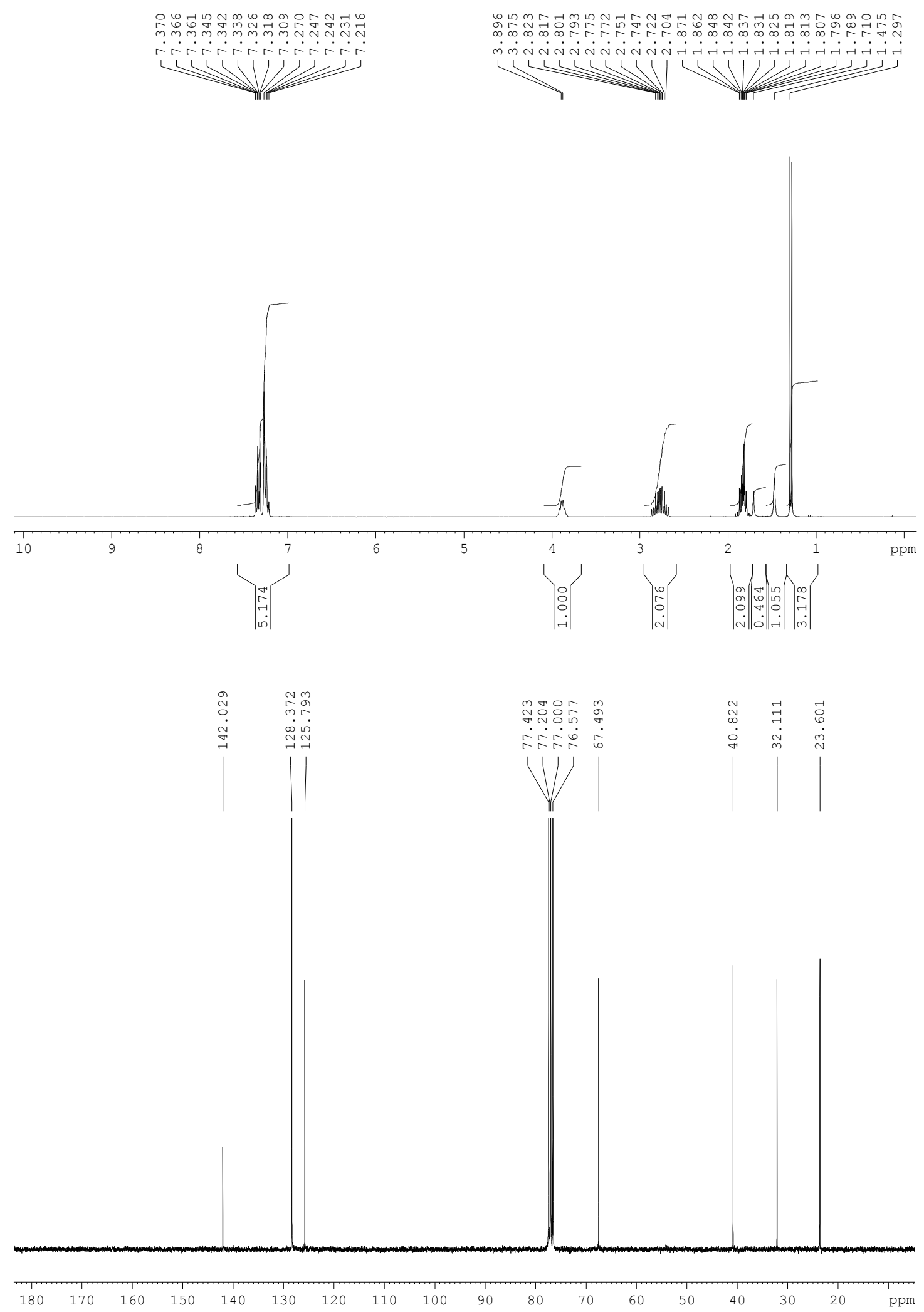




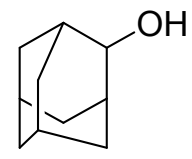

Entry 7, Table 2

(crude ${ }^{1} \mathrm{H}$ NMR spectra, contains adamantan-2-ol and $\gamma$-butyrolactone)

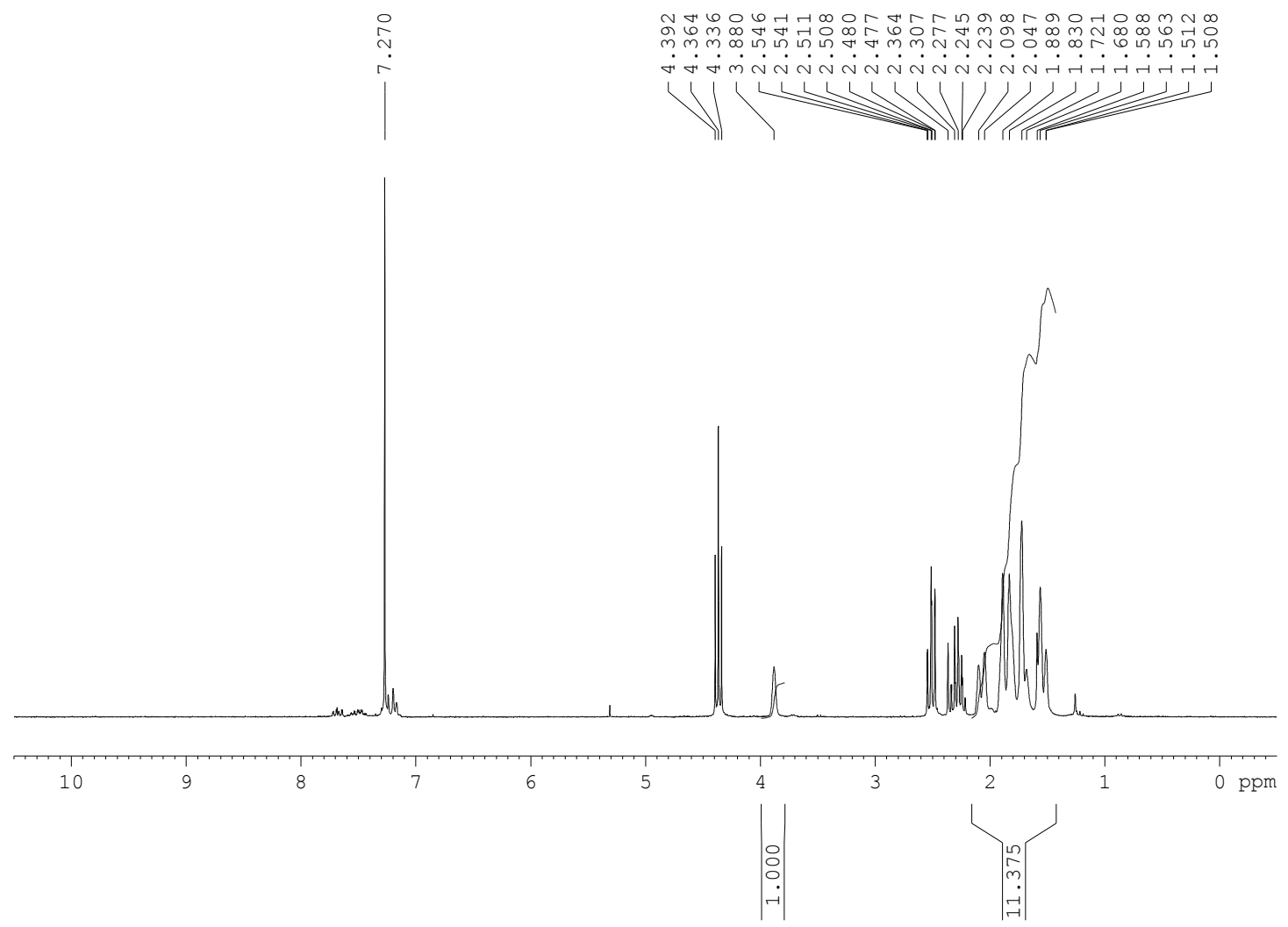


<smiles>OC1CCCCC1</smiles>

Entry 8, Table 2

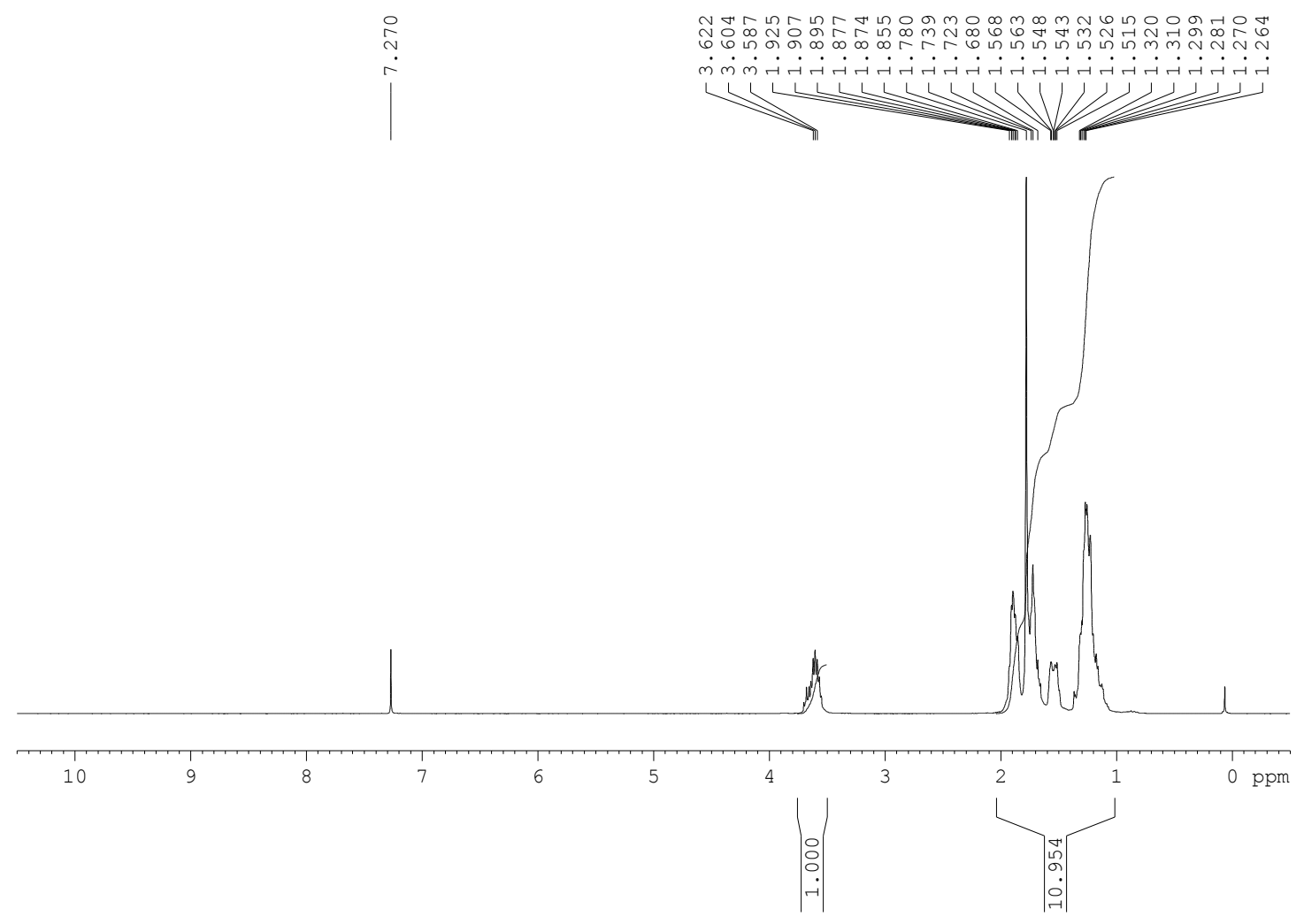


<smiles>OCc1ccccc1</smiles>

Entry 9, Table 2
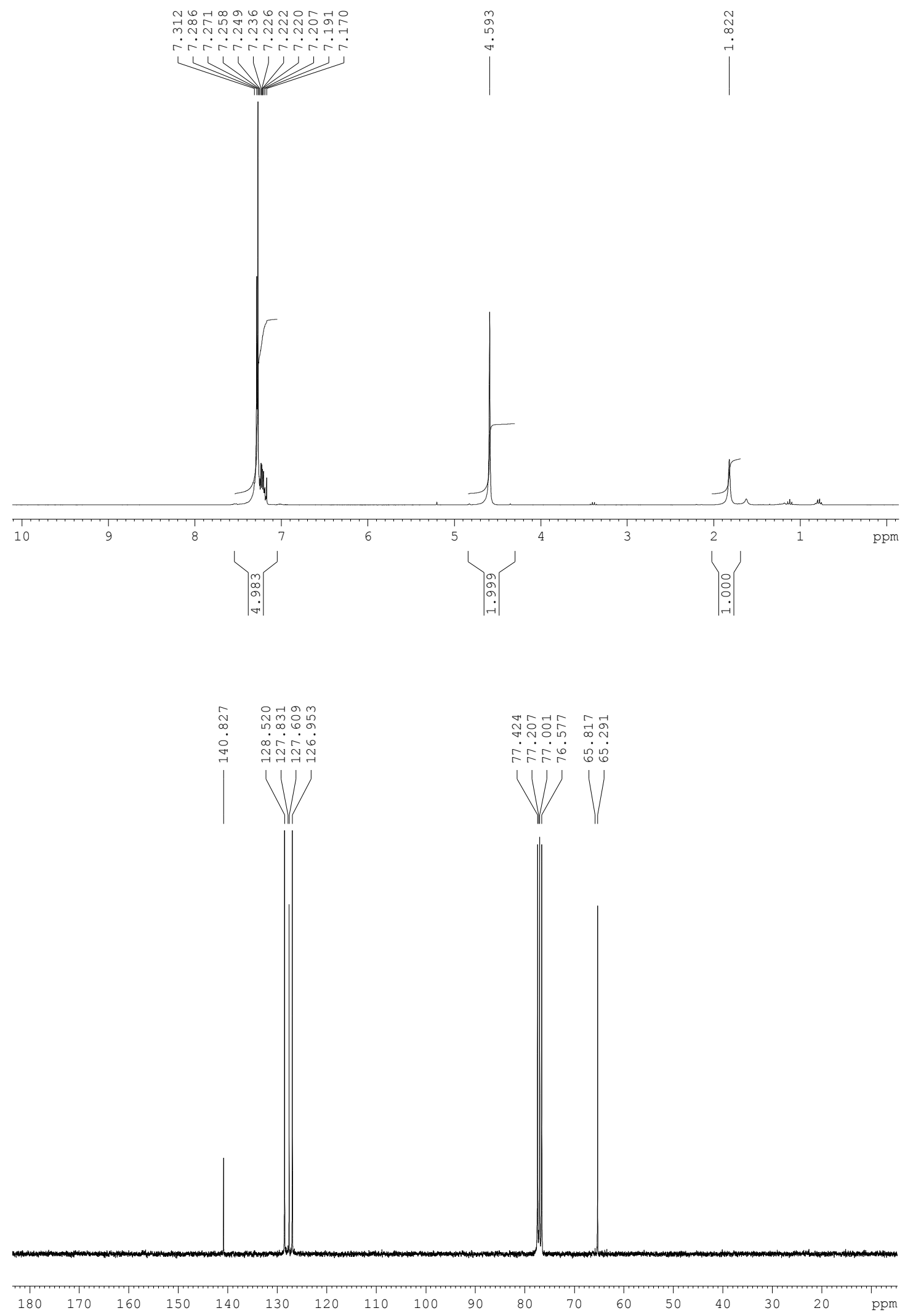


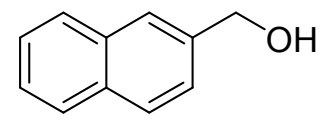

\section{Entry 10, Table 2}

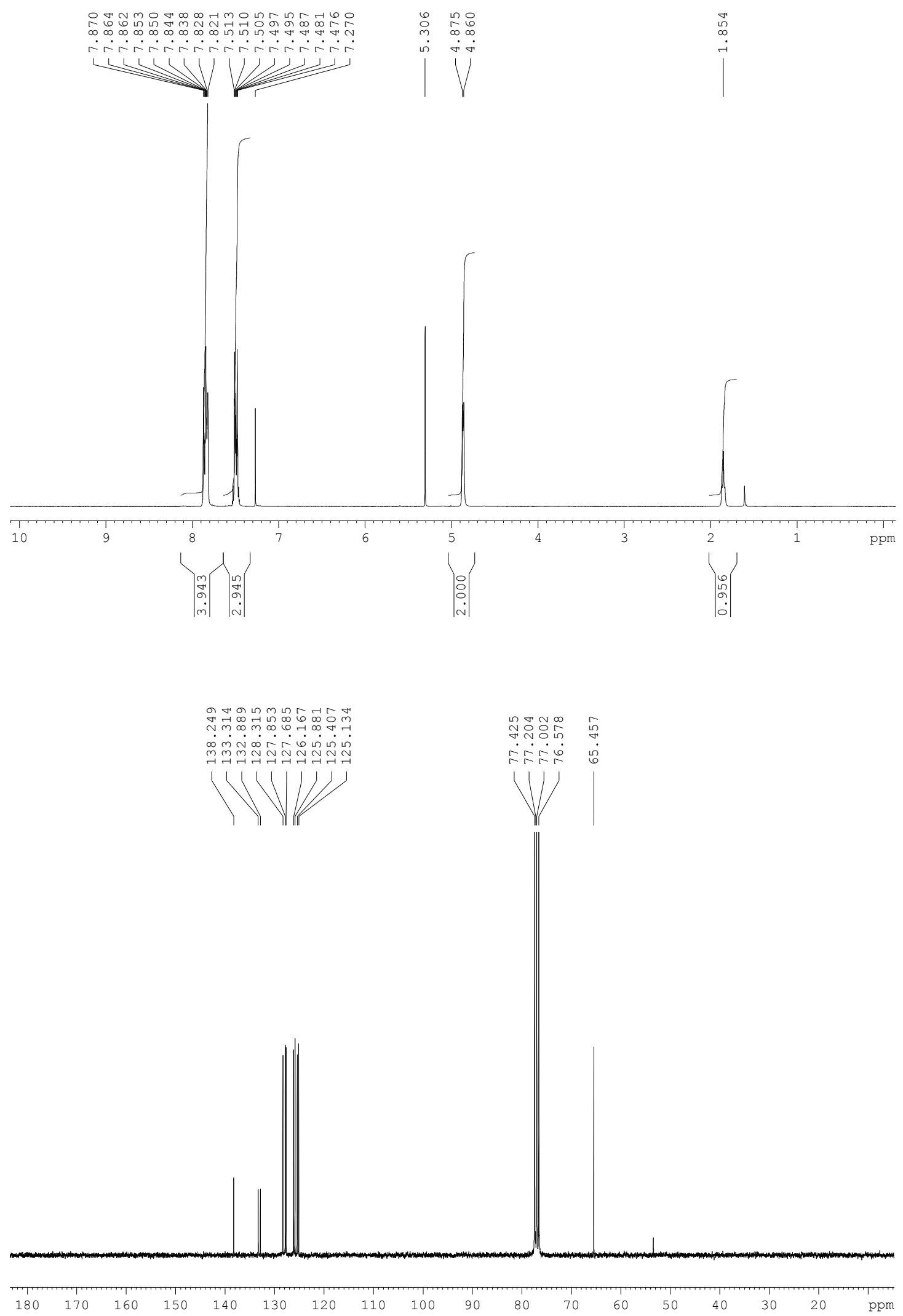




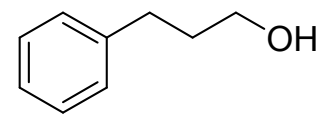

\section{Entry 11, Table 2}

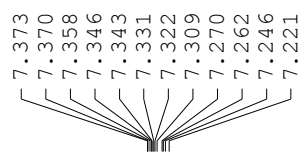

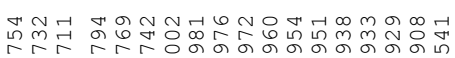

लंभ लंखेल

v lulvill
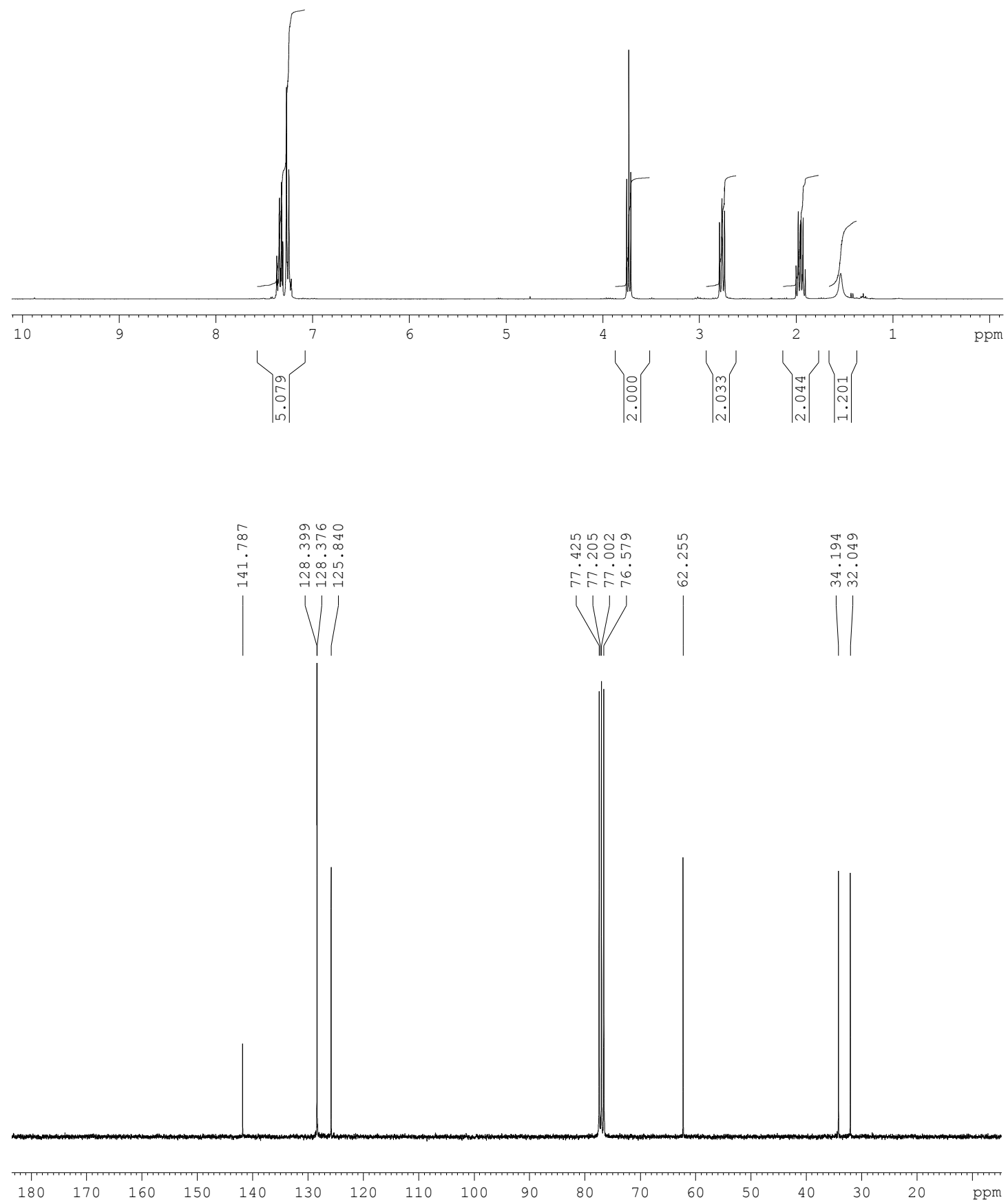
${ }^{1}$ H NMR spectrum for the combined use of 1,4-butanediol with methyl levulinate

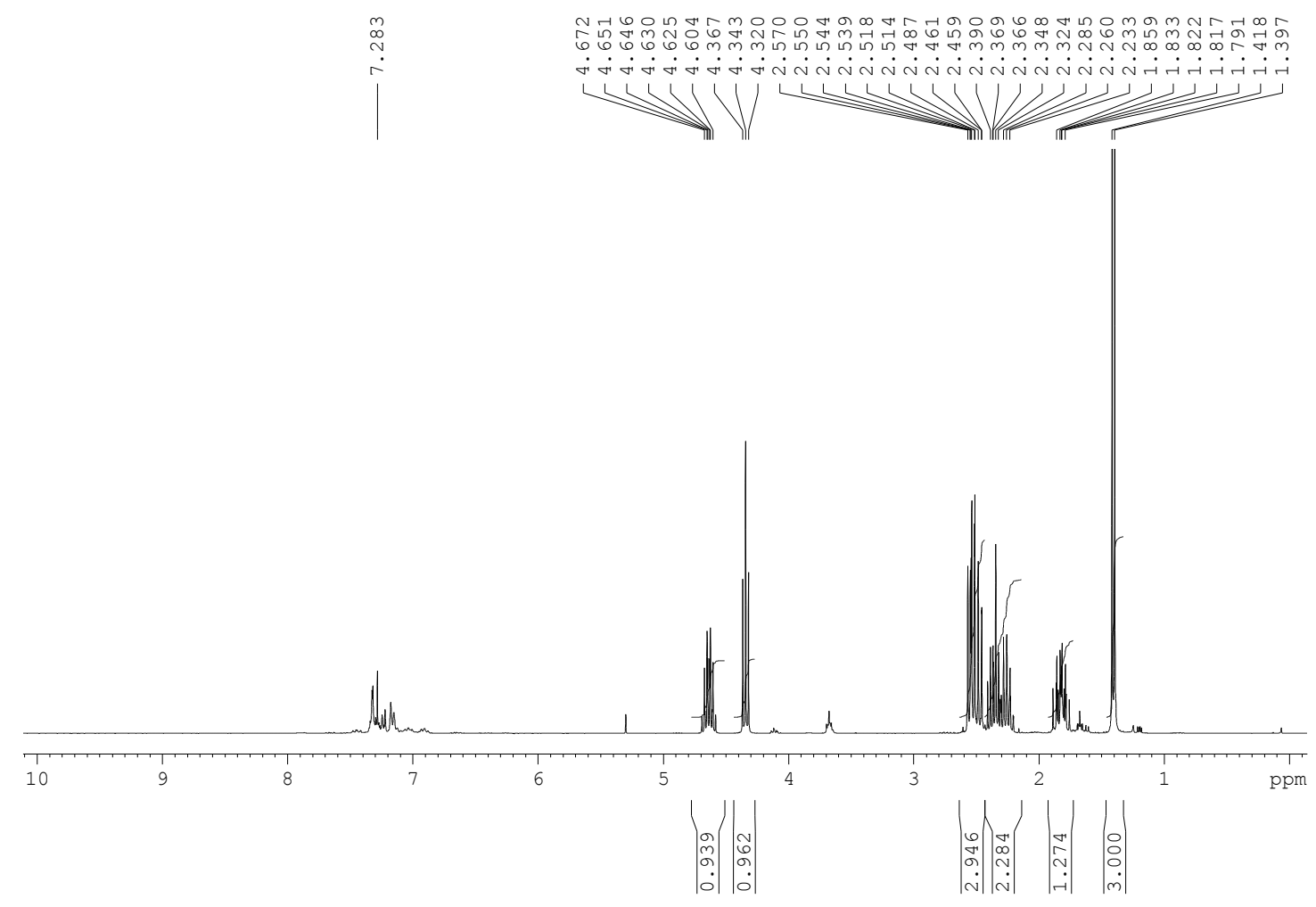

